\title{
Lipid Droplets Accumulate in the Hypothalamus of Mice and Humans with and without Metabolic Diseases
}

\author{
Clarissa Menezes Maya-Monteiro ${ }^{a, b, c}$ Felipe Corrêa-da-Silva ${ }^{b}$ \\ Susanna S. Hofmann ${ }^{d}$ Matthijs K.C. Hesselink ${ }^{e}$ Susanne E. la Fleur ${ }^{b, c}$ \\ Chun-Xia Yi ${ }^{b}$ \\ a Laboratory of Immunopharmacology, Instituto Oswaldo Cruz, Fundação Oswaldo Cruz (FIOCRUZ), \\ Rio de Janeiro, Brazil; ' Laboratory of Endocrinology and Department of Endocrinology and Metabolism, \\ Amsterdam Neuroscience, Amsterdam University Medical Centers (UMC), University of Amsterdam, \\ Amsterdam, The Netherlands; ${ }^{\mathrm{C}}$ Netherlands Institute for Neuroscience, an Institute of the Royal Netherlands \\ Academy of Arts and Sciences, Amsterdam, The Netherlands; ${ }^{\mathrm{d}}$ Institute for Diabetes and Regeneration, Helmholtz \\ Diabetes Center, Helmholtz Zentrum München, German Research Center for Environmental Health (GmbH), \\ Neuherberg, Germany; ${ }^{\text {}}$ Department of Nutrition and Movement Sciences, Maastricht University Medical Centre+ \\ and NUTRIM School for Nutrition and Translational Research in Metabolism, Maastricht, The Netherlands
}

\section{Keywords}

Lipid droplet · PLIN2 - Insulin resistance · Type 2 diabetes mellitus · Hypothalamus

\begin{abstract}
Background: In peripheral tissues, the lipid droplet (LD) organelle links lipid metabolism, inflammation, and insulin resistance. Little is known about the brain LDs. Objectives: We hypothesized that hypothalamic LDs would be altered in metabolic diseases. Methods: We used immunofluorescence labeling of the specific LD protein, PLIN2, as the approach to visualize and quantify LDs. Results: LDs were abundant in the hypothalamic third ventricle wall layer with similar heterogeneous distributions between control mice and humans. The LD content was enhanced by high-fat diet (HFD) in both wild-type and in low-density lipoprotein receptor deficient (Ldlr-/- HFD) mice. Strikingly, we observed a lower LD amount in type 2 diabetes mellitus (T2DM) patients when compared with non-T2DM patients. Conclu-
\end{abstract}

karger@karger.com www.karger.com/nen

Karger $\stackrel{\text { ' }}{5}$

GOPEN ACCESS
(C) 2020 The Author(s)

Published by S. Karger AG, Basel

This article is licensed under the Creative Commons AttributionNonCommercial-NoDerivatives 4.0 International License (CC BYNC-ND) (http://www.karger.com/Services/OpenAccessLicense) Usage and distribution for commercial purposes as well as any distribution of modified material requires written permission. sions: LDs accumulate in the normal hypothalamus, with similar distributions in human and mouse. Moreover, metabolic diseases differently modify LD content in mouse and human. Our results suggest that hypothalamic LD accumulation is an important target to the study of metabolism.

(c) 2020 The Author(s)

Published by S. Karger AG, Basel

\section{Introduction}

The development of metabolic diseases, such as obesity and type 2 diabetes mellitus (T2DM), coincides with disturbed fatty acid metabolism and excess ectopic fat storage as lipid droplets (LDs) in many peripheral tissues $[1,2]$. While LDs are linked to insulin resistance in peripheral tissues, little is known about the role of LDs in

Clarissa Menezes Maya-Monteiro

Laboratory of Immunopharmacology, Oswaldo Cruz Institute Oswaldo Cruz Fundation (FIOCRUZ)

Av. Brasil 4365, Pavilhão 108, sala 48, Rio de Janeiro 21045-900 (Brazil)

clarissa@ioc.fiocruz.br; c.maya@ amsterdamumc.nl

Chun-Xia Yi

Department of Endocrinology and Metabolism

Amsterdam University Medical Centers, location AMC, University of Amsterdam

Meibergdreef 9, NL-1105 AZ, Amsterdam (The Netherlands)

c.yi@amsterdamumc.nl 
the brain and their putative role in the pathogenesis of metabolic disease and insulin resistance [2]. The impairment of glucose uptake induced by insulin in peripheral tissues is a core feature of T2DM, and insulin resistance also occurs in the central nervous system [3-5]. Insulin resistance, induced by high-fat diet (HFD) feeding, is accompanied by disruption of lipid metabolism with ectopic lipid deposition in intracellular LDs [6-9].

LDs are hydrophobic organelles formed in the majority of cells with a core of neutral lipids and a great variety of LD proteins that are related to lipid metabolism, cell signaling, and production of inflammatory mediators [10-12]. The perilipin (PLIN) family comprises specific LD structural proteins [13], and the most studied proteins are the adipocyte PLIN1 and the PLIN2 [14]. PLIN2 is present in all cells and tissues and is exclusively present in LDs, being considered the standard marker for this organelle [15].

In Drosophila, LDs were identified in microglia and shown important for the neuronal energy supply [16]. PLIN2 has also been described in LDs in isolated mammalian brain microglia under LPS stimuli $[17,18]$. Microglia in the hypothalamus has been shown to be involved in the development of insulin resistance and lipid metabolism [19]; however, little is known about the role of other hypothalamic cell types, such as ependimocytes and tanycytes, within he hypothalamic third ventricle $(3 \mathrm{~V})$ region. These cells have barrier functions and are also important for the control of lipid and glucose metabolism/sensing [20-23], but whether LDs are present in the $3 \mathrm{~V}$ wall cells remains unclear.

In the current study, we use the PLIN2 immunoreactivity as a marker for describing the physiological and pathological presence and distribution of LDs along the $3 \mathrm{~V}$ in mouse and human with and without metabolic disorders.

\section{Materials and Methods}

\section{Mice}

C57BL/6 wild-type (WT) and low-density lipoprotein receptor deficient (Ldlr -/-) mice (Jackson Laboratories, Bar Harbor, ME, USA) were housed in a pathogen-free environment, in conformity with institutional guidelines for animal experiments and approved by the Institutional Animal Care and Use Committee at the University of Cincinnati. For the description of the normal LD distribution, we used C57BL/ 6 mice (age 8 weeks) fed with chow diet. The WT mice (age 8 weeks) were divided into 2 groups, one fed with chow diet, one with HFD containing high carbohydrates (D12079B Western diet, Research Diets, New Brunswick, NJ, USA, containing $17 \mathrm{kcal} \%$ protein, $42 \%$ carbohydrate, and $41 \mathrm{kcal} \%$ fat as well as a high-fructose corn syrup sweetened beverage) for 32 weeks. Ldlr -/- mice (age 8 weeks) were fed with the HFD for 26 weeks. All mice were housed on a 12-hour-light/12-hour-dark cycle at $22 \pm 2{ }^{\circ} \mathrm{C}$ with food and water ad libitum. At the end of the study, all mice were decapitated and brains were immerse-fixed in $4 \%$ paraformaldehyde $0.1 \mathrm{M}$ phosphate-buffered saline (PBS, $\mathrm{pH}$ 7.4) at $4{ }^{\circ} \mathrm{C}$ for $48 \mathrm{~h}$. Brains were then equilibrated $48 \mathrm{~h}$ in $30 \%$ sucrose in $0.1 \mathrm{M}$ Tris-buffered saline (TBS, pH 7.2), and coronally cut by a cryostat into $30-\mu \mathrm{m}$ sections.

\section{Human Brain Tissue}

Postmortem hypothalamic tissues of 17 T2DM and 8 nonT2DM age-matched controls and 9 non-T2DM young subjects were obtained from the Netherlands Brain Bank, through autopsy approved by the Medical Ethics Committee of the VU Medical Center, The Netherlands. Subjects who had Braak stage V-VI or clinically diagnosed severe dementia were excluded. Subject details, including sex, age, postmortem delay, clinical diagnosis, and cause of death, are provided in Table 1 and online supplementary Table 1 (see www.karger.com/doi/10.1159/000508735 for all online suppl. material). The tissues were immersion fixed in $10 \%$ phosphate-buffered formalin, then paraffin embedded and coronally sectioned in 6- $\mu \mathrm{m}$ slices. Every 100th section was used for Nissl staining to predetermine the anatomical orientation of the infundibular nucleus, which was further analyzed by the range of NPY-positive neurons. For immunofluorescence, 2 sections of the infundibular nucleus areas were mounted on SuperFrost Plus slides and dried at $37^{\circ} \mathrm{C}$. Sections were deparaffinized and hydrated, rinsed in $50 \mathrm{mM}$ TBS ( $\mathrm{pH} 7.6$ ), and were antigen retrieved for $10 \mathrm{~min}$ using microwave treatment at $700 \mathrm{~W}$ in $0.1 \mathrm{M}$ sodium citrate ( $\mathrm{pH}$ 6.0).

\section{Immunofluorescence}

Samples were washed $(2 \times)$ with TBS before incubations. All antibodies and streptavidin conjugates were diluted in TBS buffered solution containing $0.25 \%$ gelatin and $0.5 \%$ Triton $\times 100$, and all washes between incubations were done with TBS ( $4 \times ; 5 \mathrm{~min})$. In all experiments, control stainings were performed without the specific primary antibody. Samples were incubated with a guinea pig polyclonal anti-PLIN2 antibody (N-terminus aa 1-29, PROGEN, cat. GP40, 1:400 in mouse sections, 1:200 in human sections) and rabbit polyclonal anti-GFAP (DAKO, cat. Z0334, 1:100 in mouse sections, 1:500 in human sections) overnight at $4{ }^{\circ} \mathrm{C}$. Sections were then incubated for $1 \mathrm{~h}$ with a secondary anti-guinea pig conjugated with biotin antibody (Vector, cat. BA7000, 1:400). After that, sections were incubated for $3 \mathrm{~h}$ with Streptavidin-Alexa 488 (Thermo-Fisher, cat. S32354, 1:300) in the human slides, or Streptavidin-Alexa 647 (Thermo-Fisher, cat. S32357, 1:500) in the mouse free-floating slices. For the mouse sections, the Alexa-488 conjugated secondary anti-rabbit antibody (Thermo-Fisher, cat. A21206, 1:500) was added to the incubation, on the last hour. The sections were washed and incubated with DAPI (Thermo Fisher, cat. 62247, 1:2,000) and NeuroTrace Red (when indicated; Thermo Fisher, N21482) for $10 \mathrm{~min}$. After final washing, all slides were then mounted with Vectashield Hard-Set mounting media (Vector).

\section{Image Acquisition and Analysis}

Images were acquired using a TCS SP8 Confocal in DMi8 inverted microscope (Leica Microsystems, Wetzlar, Germany). Identical hardware and software settings were used for each of the 
Table 1. Clinicopathological data of patients and matched controls

\begin{tabular}{|c|c|c|c|c|c|c|}
\hline NBB No. & Sex & Age & Brk & PMD & BMI & Cause of death and clinical diagnosis \\
\hline \multicolumn{7}{|l|}{ Controls } \\
\hline 1980-009 & $\mathrm{F}$ & 72 & l & 9.0 & 23 & Epithelial carcinoma \\
\hline 1991-205 & $\mathrm{F}$ & 65 & l & 9.5 & 20 & Breast tumor, cardiac failure \\
\hline 2007-088 & $\mathrm{F}$ & 82 & 3 & 5.2 & 22 & Cachexia, cardiac failure, encephalopathy, mitral valve insufficiency \\
\hline 2009-022 & $\mathrm{F}$ & 77 & 1 & 2.9 & 33 & Pulmonary metastasis of vulva carcinoma \\
\hline 2011-082 & $\mathrm{F}$ & 84 & 2 & 5.9 & 39 & Respiratory failure, angina pectoris, mitral valve insufficiency \\
\hline 2012-005 & $\mathrm{F}$ & 84 & 2 & 5.6 & 31 & Heart failure, metastatic breast cancer, scoliosis \\
\hline 2012-033 & $\mathrm{F}$ & 95 & 3 & 5.7 & 28 & Heart failure, cachexia and dehydration, pulmonary disease \\
\hline 2012-104 & M & 79 & 2 & 6.5 & 31 & Legal euthanasia, ischemic colitis, heart failure with dyspnea \\
\hline \multicolumn{7}{|l|}{$T 2 D M$} \\
\hline 1989-032 & M & 84 & l & 4.1 & / & Heart failure, intestinal tumor \\
\hline 1995-008 & $\mathrm{F}$ & 79 & 3 & 2.1 & / & Dehydration, endometrium carcinoma \\
\hline 1997-088 & $\mathrm{F}$ & 78 & 1 & 4.3 & / & Pneumonia, seizures, CVA, cortical dysarthria syndrome \\
\hline $1998-080$ & $\mathrm{~F}$ & 72 & 3 & 24.0 & / & Respiratory failure, pulmonary hypertension \\
\hline 1998-126 & M & 71 & 2 & 6.0 & 26 & Respiratory insufficiency, lung carcinoma \\
\hline 1999-015 & $\mathrm{F}$ & 93 & 3 & 2.6 & 1 & Pneumonia, dehydration, breast cancer \\
\hline 2001-003 & M & 67 & l & 10.0 & / & Heart failure, urinary tract infection \\
\hline 2003-054 & M & 67 & 1 & 4.5 & l & Cardiac shock CVA \\
\hline 2006-033 & $\mathrm{M}$ & 79 & 1 & 5.0 & / & Pneumonia, dehydration, CVA, choledochus carcinoma \\
\hline 2008-105 & $\mathrm{F}$ & 89 & 3 & 3.9 & 30 & Pneumonia, coronary artery bypass, atrial fibrillation \\
\hline 2009-091 & M & 84 & 1 & 7.3 & 32 & Anemia, colon and prostate carcinoma metastasis \\
\hline 2010-092 & M & 86 & 3 & 5.1 & 16 & Dehydration and cachexia, CVA \\
\hline 2011-027 & M & 80 & 1 & 3.3 & 24 & Pneumonia, CVA, ischemic attack \\
\hline 2012-049 & $\mathrm{F}$ & 70 & 2 & 7.6 & / & Cachexia, pancreatic carcinoma \\
\hline 2012-088 & $\mathrm{F}$ & 85 & 3 & 6.4 & 29 & Legal euthanasia, hypoparathyroidism \\
\hline 2012-092 & M & 90 & 2 & 5.8 & / & Prostate carcinoma, CVA \\
\hline 2014-040 & M & 85 & 3 & 5.2 & / & Pulmonary carcinoma, pneumonia, arteritis temporalis \\
\hline
\end{tabular}

NBB, Netherlands Brain Bank; Brk, Braak stage; PMD, postmortem delay between time of death and time of autopsy (hours); BMI, body mass index; CVA, cerebrovascular accident.

mouse experiments and another fixed setting for the human samples.

The quantification of the mouse LDs by the PLIN2 immunoreactivity (PLIN2-ir) was performed with the Imaris software (Oxford Instruments, Abingdon, UK). Images for quantification ( $z$ stack, $20 \mu \mathrm{m}$ ) were taken with a $63 \times$ lens. The DAPI and the PLIN2-Alexa 647 images were used to generate surfaces corresponding to the total volume of nuclei and LDs, respectively, with fixed settings of threshold. The amount of LDs per nuclei was obtained by dividing the volume of PLIN2 labeling per volume of DAPI labeling. The GFAP labeling was used as a marker of astrocytes and specific a tanycytes.

The human PLIN2-ir LD quantification was done in images obtained with a $10 \times$ objective to cover half of the ventricle wall (left or right) on the slide, and 6-10 consecutive images were taken depending on the ventricle length. The analysis was made with the use of the ImageJ software and a macro developed by Ard Jonker (Cellular Imaging - Core Facility, Amsterdam UMC, location AMC). LD measurements were performed using an automatically generated stripe comprising the whole ependymal layer of the $3 \mathrm{~V}$ half wall of each patient. The total length (from sulcus to infundibulus) was divided into 10 subsections to allow comparisons between pa- tients. The region of interest to measure LD signal in the ependymal layer cells was determined by the nuclei (DAPI) staining, as detailed in the supplementary text file. The tissue fluorescence intensity outside the signal area was considered as the background signal and was subtracted from the signal fluorescence. The corrected signal of LD at the area of fluorescence was divided by the length of each subsection of the ventricle wall to give the LD fluorescence per $\mu \mathrm{m}$ of ventricle wall. The graphs and statistic calculations were performed with the GraphPad Prism software, and we used the Welch $t$ test for the comparison between specific groups.

\section{Results}

\section{Heterogeneous Distribution of LDs in Mouse $3 \mathrm{~V}$}

The presence and distribution of LDs along the $3 \mathrm{~V}$ wall in the naïve mouse hypothalamus was analyzed by the presence of PLIN2-ir in 3 regions determined by the distance to bregma along the rostral-caudal axis [24]. We observed that the majority of LDs were concentrated 


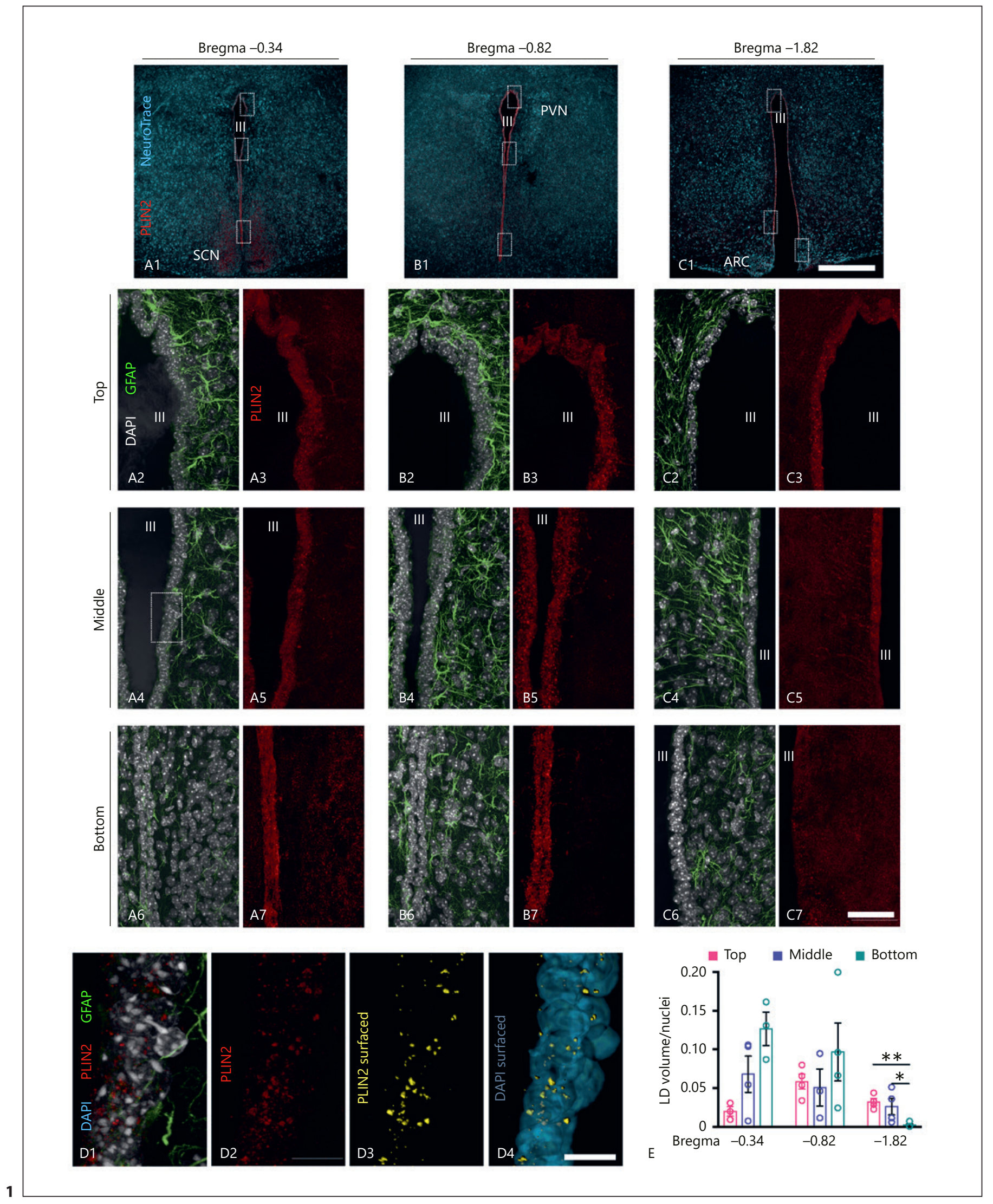

(For legend see next page.) 
along the ependymal layer of the $3 \mathrm{~V}$ in 3 different levels along the rostral-caudal axis with a heterogeneous distribution (Fig. 1). We compared the amount of LD at 3 different rostral-caudal levels, corresponding to specific surrounding hypothalamic nuclei, the suprachiasmatic (SCN; Fig. 1A), periventricular (PVN; Fig. 1B), and arcuate nuclei (ARC; Fig. 1C), and the 3 regions of the ventricle (i.e., top, middle, and bottom). Astrocytes (GFAPir) can be observed outside the ependymal layer (Fig. 1A27, B2-7, C2-7), but the majority of the astrocytes do not present LDs. At the level of bregma -1.8, the a tanycytes that project to the dorsal ARC and also express GFAP [25] coincide with an area with high amount of LDs (Fig. 1C4, C5). There is no significant variation between the different bregma levels when we compare the top and the middle regions. Interestingly, in the bottom region with GFAP-negative $\alpha 2$ tanycytes, known to project to the ventromedial $\mathrm{ARC}$, we found negligible amounts of $\mathrm{LD}$ along the ventricle wall (Fig. 1C6, C7, E).

\section{LDs in the $3 V$ Ventricle in Insulin-Resistant HFD-Fed Mice}

The ARC is a key brain region containing important neuron populations that are involved in the development of insulin resistance, and the tanycytes in this area of the $3 \mathrm{~V}$ are involved in the control of systemic energy metabolism [26]. We investigated whether the LD heterogeneous distribution at the level of bregma -1.82 of the $3 \mathrm{~V}$ was associated with the presence of insulin resistance. We studied the alterations induced by a HFD in the LD distribution in both WT mice and in the $L d l r-/-$ mice. The

Fig. 1. Distribution of LDs in normal mouse hypothalamus. Representative immunofluorescence images of the 3 different Bregma levels of hypothalamus with labelled neurons (NeuroTrace, in cyan, evidencing the hypothalamic nuclei) and LDs (LD; perilipin 2 immunoreactive, PLIN2-ir, in red; A1, B1, C1). Higher magnification $z$-stack images were obtained at the marked areas, with the additional label for cell nuclei (DAPI, in grey), glial fibrillary acidic protein-immunoreactive (GFAP-ir) astrocytes and GFAP-ir a tanycytes (in green), and LD (PLIN2-ir, in red; panels 2-7 in A, B, and $\mathbf{C})$. LDs were observed at the GFAP-ir a tanycytes region (C4). Quantification of the volume of LDs and nuclei are performed by generating the $3 \mathrm{D}$ surfaces, from the high-magnification $z$-stack images, with the IMARIS software (D). Representative image from the outlined area in A4 (original image; D1, D2; nuclei in grey; LD in red) (surface image; D3, D4; nuclei in cyan, LD in yellow). The volume of LDs was normalized by the volume of nuclei in order to compare the different areas (E). Scale bar, $400 \mu \mathrm{m}$ in A1, B1, C1; 40 $\mu \mathrm{m}$ in A2-A7, B2-B7, C2-C7; $10 \mu \mathrm{m}$ in D1-D4. III, third ventricle. Data are presented as mean \pm SEM of a minimum $n=3 . * p<0.05$; ** $p<0.01$ according to the Welch $t$ test.

Lipid Droplets in Hypothalamus
$L d l r-/-$ mice on a HFD present a complete phenotype for the study of metabolic diseases with enhanced adipose tissue, insulin resistance, and hypothalamic inflammation $[7,8]$. The top area of the ventricle, with a majority of ependimocytes, has enhanced LDs in both the HFDfed WT and the HFD-fed Ldlr-/- mice (Fig. 2A1, A2, B1, B2, C1, C2, E). The GFAP-ir a tanycytes area presents a high amount of LDs similarly to the top part of the ventricle in all the groups (Fig. 2A3, A4, B3, B4, C3, C4). The lower part of the ventricle wall with GFAP-negative $\alpha$ and $\beta$ tanycytes (as shown in Fig. 1) has very low amounts of LDs in the WT on chow diet (Fig. 2A5, A6, E). Moreover, the HFD feeding in the WT mice did not induce LDs in this area (Fig. 2B5, B6, E). In the HFD-fed $L d l r-/-$ mice, with a more severe metabolic disruption, we can observe enhanced LDs at the ARC level (Fig. 2C5, C6, E).

\section{LDs in the $3 \mathrm{~V}$ in Control (Non-Diabetic) and T2DM Subjects}

Insulin resistance induced by HFD in animal models does not fully mimic the human T2DM pathogenesis, and this is a major obstacle hampering translational studies on brain dysfunction in T2DM patients [19]. We therefore investigated the distribution of LDs in the human $3 \mathrm{~V}$, comparing control (non-diabetic patients) with T2DM patients. We used hypothalamic samples from the Netherlands Brain Bank selected with age matched between the groups (Table 1). We show here that the human $3 \mathrm{~V}$ wall contains significant amounts of LDs as evidenced by the PLIN2 staining (Fig. 3A) and the LDs are mainly associated with the ependimocyte layer (Fig. 3B). All the patients with different ages, distinct causes of death, different body mass index (BMI, only part of the subjects had BMI data) and different postmortem delay present a significant amount of $\mathrm{LD}$ in the $3 \mathrm{~V}$ wall. Moreover the LD content in this group of subjects did not correlate with age, BMI, or postmortem delay (online suppl. Fig. 1). It is important to point out that we also show the presence of LDs already in the $3 \mathrm{~V}$ wall from a separate group of young brains (online suppl. Fig. 3; online suppl. Table 1). To compare the control patients with the T2DM patients, we performed the quantification of the LDs along the ventricle as described in the Methods section (Fig. 3C). In the control and T2DM subjects, we found the same pattern of distribution of LD as in mice, with higher amounts of LDs in the top and middle areas (PVN and $\mathrm{DMH}$ ) and lower amounts in the bottom area (infundibular nucleus; Fig. 3A, C, D). Within the T2DM patients, this distribution is less pronounced with lower amounts of LDs in the ventricle wall cells (Fig. 3C, D, E). 


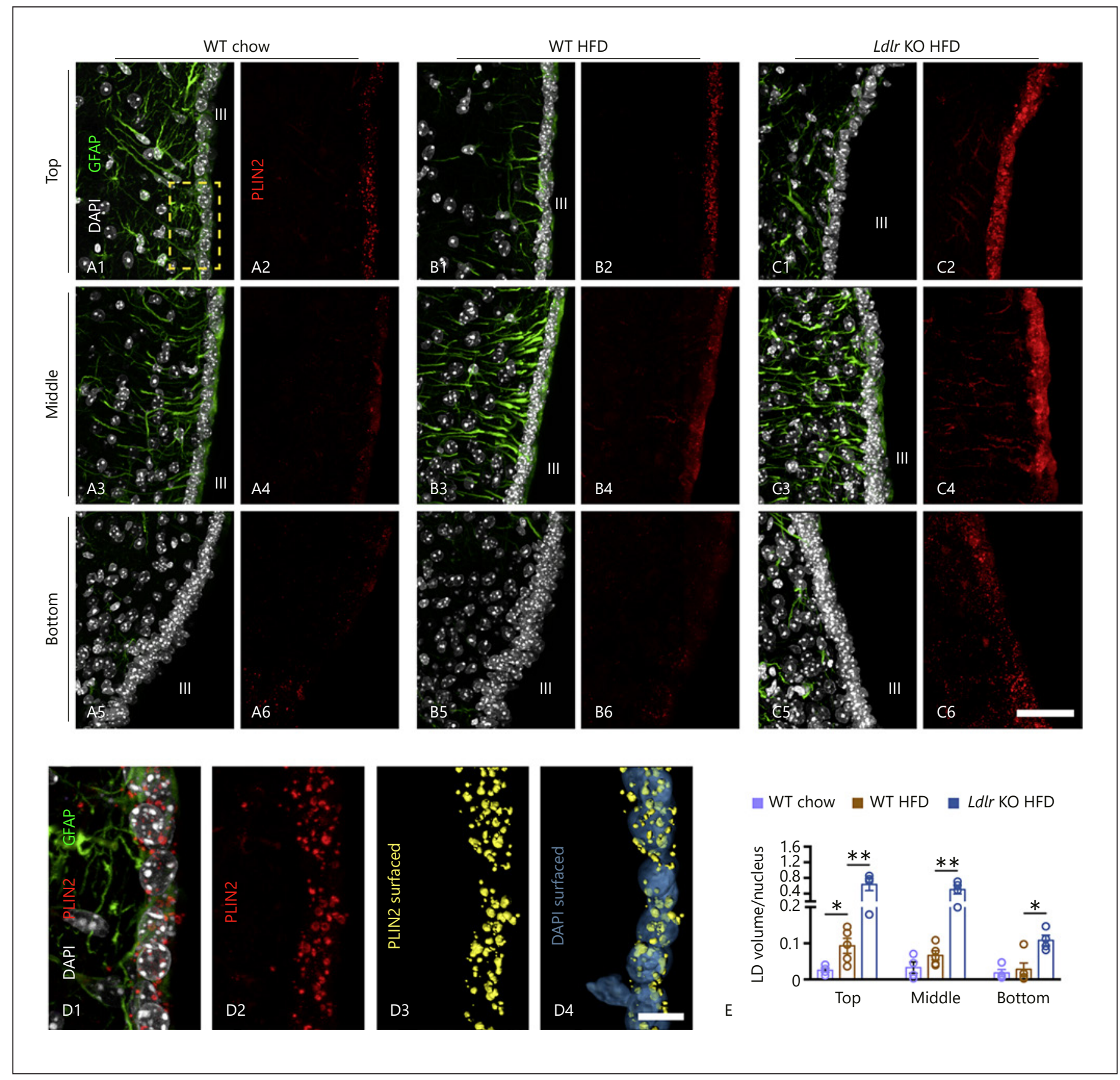

Fig. 2. LD content is altered in the hypothalamus of insulin-resistant mice. Representative immunofluorescence images of the mouse hypothalamus (bregma -1.82) from WT (A1-A6), high-fat diet (HFD)-fed WT mice (WT HFD; B1-B6), and HFD-fed lowdensity lipoprotein receptor knockout mice ( $L d l r$ KO HFD, C1C6). Cell nuclei (DAPI; in grey), glial fibrillary acidic protein-immunoreactive (GFAP-ir) astrocytes and GFAP-ir a tanycytes (in green), and LD (PLIN2-ir, in red; A, B, C). Quantification of the volume of LDs and nuclei are performed by generating the $3 \mathrm{D}$ sur- faces with the IMARIS software (D). Representative image from the outlined area in A1 (original image; D1, D2; nuclei in grey; LD in red) (surface image; D3, D4; nuclei in cyan, LD in yellow). The volume of LDs was normalized by the volume of nuclei in order to compare the different areas (E). Scale bar, $40 \mu \mathrm{m}$ in A1-C6; $10 \mu \mathrm{m}$ in D1-D4. III, third ventricle. Data are presented as mean \pm SEM of a minimum $n=4 .{ }^{*} p<0.05{ }^{* *} p<0.01$ according to the Welch $t$ test. 

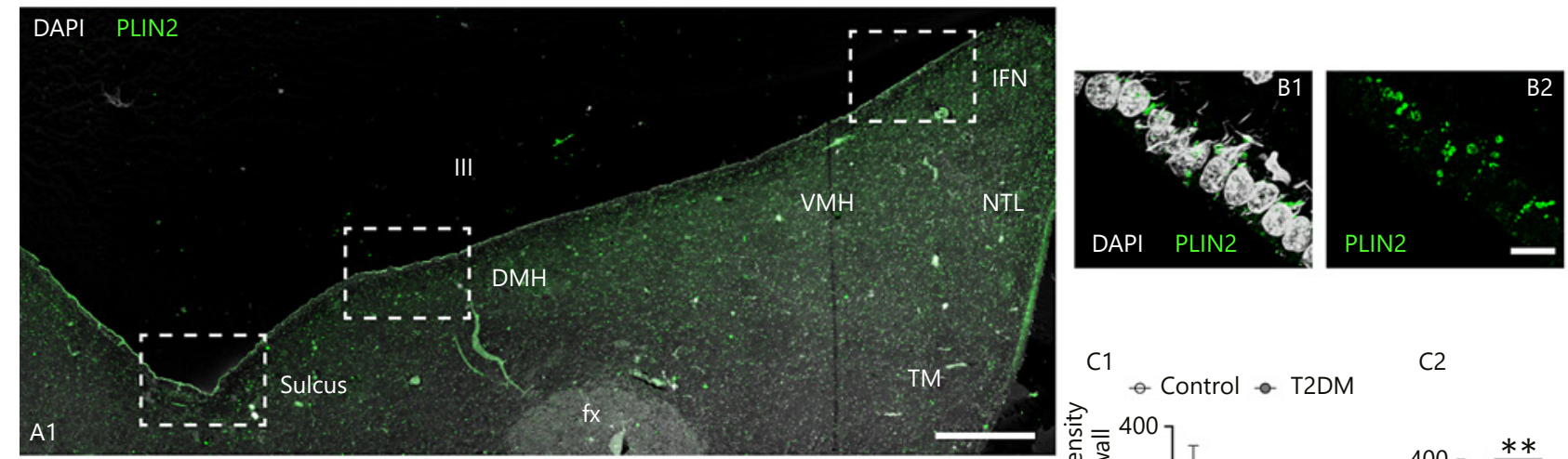

C1 $\rightarrow$ Control $-\mathrm{T}$ T2DM

C2
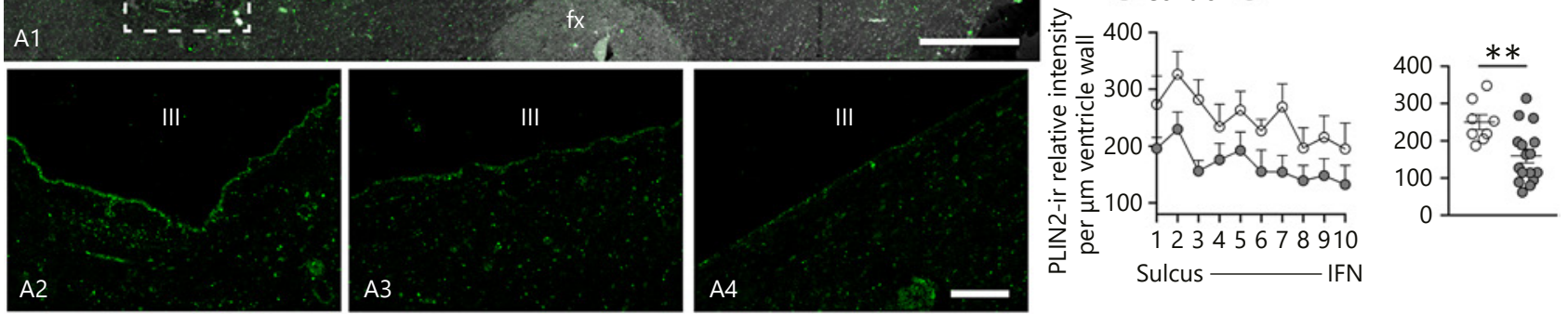

Control
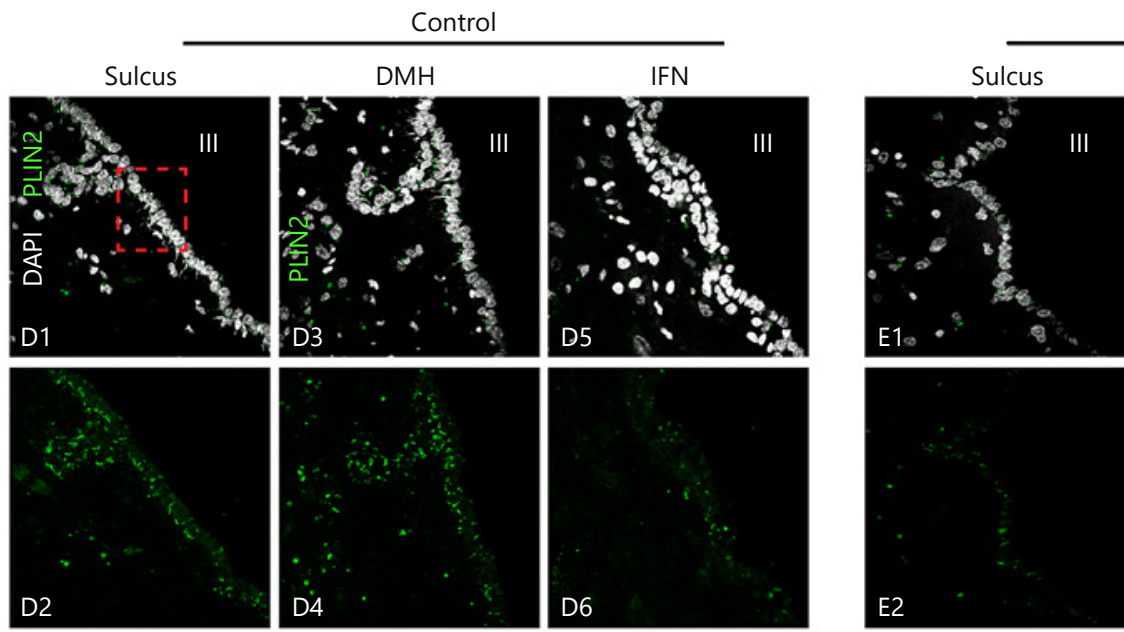

T2DM

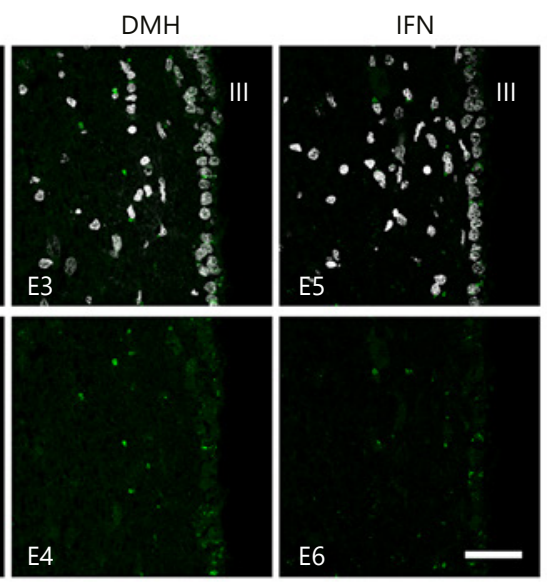

Fig. 3. Characterization of LD distribution and content in the third ventricle of non-diabetic controls and T2DM individuals. The anatomical orientation of different hypothalamic areas along the third cerebral ventricle and the distribution of perilipin $2 \mathrm{immu}-$ noreactive (PLIN2-ir, in green) LDs and cell nuclei (DAPI, in grey; A1). Higher-magnification images from 3 regions outlined in $\mathbf{A 1}$ are shown in A2-A4. The morphology of the LDs within the ependimocytes is demonstrated by images in $\mathbf{B} \mathbf{1}$ and $\mathbf{B 2}$ from the area selected in D1. The PLIN2-ir LD content was quantified as described in the Methods section from the images as in $\mathbf{A} \mathbf{1}$ and averaged for the 3 different regions of the ventricle wall according to

the hypothalamic nuclei in the adjacent parenchyma (C). Representative images with higher magnification, showing the nuclei (DAPI, grey) and LD (PLIN2-ir, green), to evidence the variation of LDs between the control and the T2DM patients (D, E). III, third ventricle lumen; $\mathrm{DMH}$, dorsomedial hypothalamic nucleus; $\mathrm{VMH}$, ventromedial hypothalamic nucleus; IFN, infundibular hypothalamic nucleus. Scale bar, $1 \mathrm{~mm}$ in $\mathbf{A} 1 ; 200 \mu \mathrm{m}$ in A2-A4; $10 \mu \mathrm{m}$ in B1-B2; $40 \mu \mathrm{m}$ in D1-D6, E1-E6. Data are presented as mean \pm SEM of $n=8$ (controls) and $n=17$ (T2DM). $* p<0.05$ according to the Welch $t$ test. 


\section{Discussion}

Here we provide evidence for the existence of significant amounts of LD in normal hypothalami in both mouse and human, concentrated in the wall of the $3 \mathrm{~V}$. We further demonstrate that with high fat feeding and genetic deletion of LDL receptor in mice, the LD content is augmented, depending on the degree of metabolic disturbances. Interestingly, in the human brain, the presence of T2DM disease was accompanied by lower amounts of LDs in the hypothalamus. Taken together, our data point to an important role for brain LDs in metabolism.

To our knowledge, we are the first to show a major $\mathrm{LD}$ accumulation within the ependymal layer of the $3 \mathrm{~V}$ both in physiological and pathological conditions. Earlier studies have described the presence of LDs in the brain ventricle ependymal layer; however, they only identified significant LDs in the diseased brain with Alzheimer disease or ageing [27-29]. These authors propose that the presence of LDs in the brain is correlated directly to a pathological condition. We could demonstrate the age correlation when the group of younger subjects was analyzed together with the older non-T2DM control group (online suppl. Fig. 3). Our findings point to a broader physiological role for the accumulation of LDs along the ventricle, which is also modified in metabolic diseases and ageing. The heterogeneity of the distribution of LDs along the $3 \mathrm{~V}$ is evident in the mouse and human brains and this pattern is specifically modified under pathogenic conditions. Interestingly, the changes in LDs in mouse brain with metabolic disruption either with HF feeding alone, or even more severe, through Ldlr deletion, was region specific. We found that the more metabolically disturbed, the more markedly the increase of LDs in the top of the ventricle, whereas there were discrete increases in $\mathrm{LD}$ in the $\mathrm{ARC}$ region of the $3 \mathrm{~V}$ wall. Interestingly, in humans with T2DM, we observed a reduction of LDs throughout the ventricle regions. We can speculate that the complexity and chronic conditions of human T2DM may lead to a reduction of LDs when compared to the control, non-diabetic patients.

LD organelles present a hydrophobic core of neutral lipids that can be partially or completely extracted during fixation procedures. This characteristic makes it difficult to fully assess the presence of LDs in the brain by using hydrophobic probes solely. PLIN2 is the standard and very specific marker of LDs in all cells and the protein is readily degraded when not associated with the organelle [30]. LDs have distinct roles depending on the cell and stimuli. The most studied function is of lipid storage or- ganelles, but LDs are also known sites of inflammatory mediator production and intracellular signaling and vary in protein and lipid composition and size $[31,32]$. We observed a great diversity of LD size $(0.5-4 \mu \mathrm{m})$ in the ependymal layer. According to the literature, this suggests diverse roles for these LDs in lipid storage (larger) and in control of lipid metabolism, inflammation, and signaling (smaller) [32, 33].

A previous study proposed that only the tanycytes, and not the ependymal cells, would accumulate LDs only on HFD [20]. However, we show here that the majority of LDs in both mouse and in human brain is clearly associated with the ependimocytes, and there is a high content of LDs in areas with no tanycytes. These results show that ependimocytes and tanycytes accumulate LDs and may have a physiological role in brain lipid metabolism.

Recently, Geller et al. [34] showed that mouse tanycytes can sense palmitate, in an oxidation-dependent manner, and modulate neurons to indirectly inhibit the lipolysis in the periphery. Interestingly, these palmitatesensing tanycytes are exactly in the region of the GFAPnegative $\alpha$ tanycytes that we show here. Our suggestion is that this ARC region of the ventricle is a specialized lipidsensing area and, therefore, does not physiologically accumulate LDs. Additionally, we suggest that this is an important feature since the same pattern was present in both mouse and human hypothalami.

Earlier reports showed LD accumulation in microglia and astrocytes being important in the control of neuronal metabolism $[16,35,36]$. In line, we also observed LDs in both microglia and astrocytes; however, less abundant when compared to the ependymal layer (as marked by Iba1-ir and GFAP-ir; online suppl. Fig. 2).

We conclude that the presence of hypothalamic LDs is region specific and is modified depending on the metabolic conditions in both mice and humans. The characterization of the distribution of the LDs opens possibilities for better understanding of lipid metabolism and lipid sensing in the brain. We propose that the evaluation of the LD organelle within the brain tissue should always be considered when studying the hypothalamic control of metabolism under physiological and pathogenic conditions.

\section{Acknowledgements}

The authors want to express their gratitude to Martin Kalsbeek, Unga Unmehopa, Khalid Lamuadni, and Nikita Korpel for laboratory technical assistance and to Ard Jonker for the development of the ImageJ macro. 


\section{Statement of Ethics}

Postmortem hypothalamic human tissues were obtained from the Netherlands Brain Bank, through autopsy approved by the Medical Ethics Committee of the VU Medical Center, The Netherlands.

\section{Conflict of Interest Statement}

The authors have no conflicts of interest to declare.

\section{Funding Sources}

This work was supported by Fundação Oswaldo Cruz (FIOCRUZ), Instituto Oswaldo Cruz (IOC) and Conselho Nacional de Desenvolvimento Científico e Tecnológico (306290/2014-6 CNPq)
(C.M.M.-M.), AMC fellowship (C.-X.Y., 2014, Amsterdam University Medical Center), the Dutch Diabetes Research Foundation (C.-X.Y., Diabetes Fonds, 2015.82.1826), by the Netherlands Organization of Scientific Research (NWO-VICI grant 016.160.617), and by the Deutsche Forschungsgemeinschaft (German Research Foundation) within the framework of the Munich Cluster for Systems Neurology (EXC 2145 SyNergy). All authors declare no competing interests.

\section{Author Contributions}

The conception of the study was done by C.M.M.-M., S.E.F., M.K.C.H., and C.-X.Y. The experimental design, execution, analysis, and interpretation of the data was done by C.M.M.-M., S.E.F., F.C.S., S.S.H., and C.-X.Y. The manuscript was written by C.M.M.M. and C.-X.Y. and the final version was critically revised and approved by all the authors.

\section{References}

1 Olofsson SO, Andersson L, Håversen L, Olsson C, Myhre S, Rutberg M, et al. The formation of lipid droplets: possible role in the development of insulin resistance/type 2 diabetes. Prostaglandins Leukot Essent Fatty Acids. 2011 Nov;85(5):215-8.

2 Gemmink A, Goodpaster BH, Schrauwen P, Hesselink MK. Intramyocellular lipid droplets and insulin sensitivity, the human perspective. Biochim Biophys Acta Mol Cell Biol Lipids. 2017 Oct;1862(10 Pt B):1242-9.

3 Lewis GF, Carpentier A, Adeli K, Giacca A. Disordered fat storage and mobilization in the pathogenesis of insulin resistance and type 2 diabetes. Endocr Rev. 2002 Apr;23(2): 201-29.

4 Kahn BB, Flier JS. Obesity and insulin resistance. J Clin Invest. 2000 Aug;106(4):473-81.

5 Steculorum SM, Solas M, Brüning JC. The paradox of neuronal insulin action and resistance in the development of aging-associated diseases. Alzheimers Dement. 2014 Feb;10(1 Suppl):S3-11.

6 Merat S, Casanada F, Sutphin M, Palinski W, Reaven PD. Western-type diets induce insulin resistance and hyperinsulinemia in LDL receptor-deficient mice but do not increase aortic atherosclerosis compared with normoinsulinemic mice in which similar plasma cholesterol levels are achieved by a fructoserich diet. Arterioscler Thromb Vasc Biol. 1999 May;19(5):1223-30.

7 Schreyer SA, Vick C, Lystig TC, Mystkowski P, LeBoeuf RC. LDL receptor but not apolipoprotein $\mathrm{E}$ deficiency increases diet-induced obesity and diabetes in mice. Am J Physiol Endocrinol Metab. 2002 Jan;282(1):E207-14.

8 Yi CX, Al-Massadi O, Donelan E, Lehti M, Weber J, Ress C, et al. Exercise protects against high-fat diet-induced hypothalamic inflammation. Physiol Behav. 2012 Jun; 106(4):485-90
9 Gupte AA, Minze LJ, Reyes M, Ren Y, Wang $X, B r u n n e r ~ G$, et al. High-fat feeding-induced hyperinsulinemia increases cardiac glucose uptake and mitochondrial function despite peripheral insulin resistance. Endocrinology. 2013 Aug;154(8):2650-62.

10 Zhang C, Liu P. The New Face of the Lipid Droplet: Lipid Droplet Proteins. Proteomics. 2019 May; 19(10):e1700223.

11 Welte MA, Gould AP. Lipid droplet functions beyond energy storage. Biochim Biophys Acta Mol Cell Biol Lipids. 2017 Oct;1862(10 Pt B):1260-72.

12 Maya-Monteiro CM, Bozza PT. Leptin and mTOR: partners in metabolism and inflammation. Cell Cycle. 2008 Jun;7(12):1713-7.

13 Brasaemle DL. Thematic review series: adipocyte biology. The perilipin family of structural lipid droplet proteins: stabilization of lipid droplets and control of lipolysis. J Lipid Res. 2007 Dec;48(12):2547-59.

14 Blanchette-Mackie EJ, Dwyer NK, Barber T, Coxey RA, Takeda T, Rondinone CM, et al. Perilipin is located on the surface layer of intracellular lipid droplets in adipocytes. J Lipid Res. 1995 Jun;36(6):1211-26.

15 Sztalryd C, Brasaemle DL. The perilipin family of lipid droplet proteins: gatekeepers of intracellular lipolysis. Biochim Biophys Acta Mol Cell Biol Lipids. 2017 Oct;1862(10 Pt B): 1221-32.

16 Liu L, MacKenzie KR, Putluri N, MaletićSavatić M, Bellen HJ. The Glia-Neuron Lactate Shuttle and Elevated ROS Promote Lipid Synthesis in Neurons and Lipid Droplet Accumulation in Glia via APOE/D. Cell Metab. 2017 Nov;26(5):719-37.e6.
17 Fan B, Dun SH, Gu JQ, Guo Y, Ikuyama S. Pycnogenol Attenuates the Release of Proinflammatory Cytokines and Expression of Perilipin 2 in Lipopolysaccharide-Stimulated Microglia in Part via Inhibition of NF- $\kappa \mathrm{B}$ and AP-1 Activation. PLoS One. 2015 Sep; 10(9):e0137837.

18 Khatchadourian A, Bourque SD, Richard VR Titorenko VI, Maysinger D. Dynamics and regulation of lipid droplet formation in lipopolysaccharide (LPS)-stimulated microglia. Biochim Biophys Acta. 2012 Apr;1821(4): $607-17$.

19 Hogenboom R, Kalsbeek MJ, Korpel NL, de Goede P, Koenen M, Buijs RM, et al. Loss of arginine vasopressin- and vasoactive intestinal polypeptide-containing neurons and glial cells in the suprachiasmatic nucleus of individuals with type 2 diabetes. Diabetologia. 2019 Nov;62(11):2088-93.

20 Hofmann K, Lamberz C, Piotrowitz K, Offermann N, But D, Scheller A, et al. Tanycytes and a differential fatty acid metabolism in the hypothalamus. Glia. 2017 Feb;65(2):231-49.

21 Frayling C, Britton R, Dale N. ATP-mediated glucosensing by hypothalamic tanycytes. J Physiol. 2011 May;589(Pt 9):2275-86

22 Gao Y, Tschöp MH, Luquet S. Hypothalamic tanycytes: gatekeepers to metabolic control. Cell Metab. 2014 Feb;19(2):173-5.

23 García-Cáceres C, Balland E, Prevot V, Luquet S, Woods SC, Koch M, et al. Role of astrocytes, microglia, and tanycytes in brain control of systemic metabolism. Nat Neurosci. 2019 Jan;22(1):7-14.

24 Paxinos G, Franklin KB. Paxinos and Franklin's the mouse brain in stereotaxic coordinates. Amsterdam [etc.]: Elsevier/Academic Press; 2013. 
25 Robins SC, Stewart I, McNay DE, Taylor V, Giachino C, Goetz M, et al. a-Tanycytes of the adult hypothalamic third ventricle include distinct populations of FGF-responsive neural progenitors. Nat Commun. 2013;4(1): 2049.

26 Goodman T, Hajihosseini MK. Hypothalamic tanycytes-masters and servants of metabolic, neuroendocrine, and neurogenic functions. Front Neurosci. 2015 Oct;9:387.

27 Hamilton LK, Fernandes KJ. Neural stem cells and adult brain fatty acid metabolism: lessons from the 3xTg model of Alzheimer's disease. Biol Cell. 2018 Jan;110(1):6-25.

28 Ogrodnik M, Zhu Y, Langhi LG, Tchkonia T, Krüger P, Fielder E, et al. Obesity-Induced Cellular Senescence Drives Anxiety and Impairs Neurogenesis. Cell Metab. 2019 May; 29(5):1061-77.e8.
29 Shimabukuro MK, Langhi LG, Cordeiro I, Brito JM, Batista CM, Mattson MP, et al. Lipid-laden cells differentially distributed in the aging brain are functionally active and correspond to distinct phenotypes. Sci Rep. 2016 Mar;6(1):23795.

30 Bickel PE, Tansey JT, Welte MA. PAT proteins, an ancient family of lipid droplet proteins that regulate cellular lipid stores. Biochim Biophys Acta. 2009 Jun;1791(6):419-40.

31 Welte MA. Expanding roles for lipid droplets. Curr Biol. 2015 Jun;25(11):R470-81.

32 Pereira-Dutra FS, Teixeira L, de Souza Costa MF, Bozza PT. Fat, fight, and beyond: the multiple roles of lipid droplets in infections and inflammation. J Leukoc Biol. 2019 Sep; 106(3):563-80.
33 Fujimoto T, Parton RG. Not just fat: the structure and function of the lipid droplet. Cold Spring Harb Perspect Biol. 2011 Mar; 3(3):a004838

34 Geller S, Arribat Y, Netzahualcoyotzi C, Lagarrigue S, Carneiro L, Zhang L, et al. Tanycytes Regulate Lipid Homeostasis by Sensing Free Fatty Acids and Signaling to Key Hypothalamic Neuronal Populations via FGF21 Secretion. Cell Metab. 2019 Oct;30(4):833-844. e7.

$35 \mathrm{Hu} \mathrm{X}, \mathrm{Xu} \mathrm{B}, \mathrm{Ge}$ W. The Role of Lipid Bodies in the Microglial Aging Process and Related Diseases. Neurochem Res. 2017 Nov;42(11): 3140-8.

36 Bélanger M, Allaman I, Magistretti PJ. Brain energy metabolism: focus on astrocyte-neuron metabolic cooperation. Cell Metab. 2011 Dec;14(6):724-38. 